\title{
What is the accuracy of sentinel lymph node biopsy for gastric cancer? A systematic review
}

\author{
Roberta Cardoso • Alina Bocicariu • Matthew Dixon • \\ Lavanya Yohanathan · Rajini Seevaratnam • Lucy Helyer • \\ Calvin Law $\cdot$ Natalie G. Coburn
}

Received: 18 August 2011/Accepted: 23 September 2011/Published online: 20 January 2012

(C) The International Gastric Cancer Association and The Japanese Gastric Cancer Association 2012

\begin{abstract}
Background In gastric cancer, the utility of sentinel lymph node (SLN) biopsy has not been established. SLN may be a good predictor of the pathological status of other lymph nodes and thus the necessity for more extensive surgery or lymph node dissection. We aimed to identify and synthesize findings on the performance of SLN biopsies in gastric cancer.

Methods Electronic literature searches were conducted using Medline, EMBASE, and the Cochrane Central Register of Controlled Trials from 1998 to 2009. Titles and abstracts were independently rated for relevance by a minimum of two reviewers. Techniques, detection rates, accuracy, sensitivity, specificity, and false-negative rates
\end{abstract}

Electronic supplementary material The online version of this article (doi:10.1007/s10120-011-0103-8) contains supplementary material, which is available to authorized users.

R. Cardoso - A. Bocicariu - M. Dixon - L. Yohanathan ·

R. Seevaratnam · C. Law · N. G. Coburn ( $₫)$

Division of Surgical Oncology, Sunnybrook Research Institute,

Odette Cancer Centre, Sunnybrook Health Sciences Centre,

Suite T2-60, 2075 Bayview Ave, Toronto, ON M4N 3M5,

Canada

e-mail: natalie.coburn@sunnybrook.ca

M. Dixon

Department of Surgery, Maimonides Medical Center,

Brooklyn, NY, USA

L. Helyer

Department of Surgery, Dalhousie University,

Halifax, NS, Canada

C. Law · N. G. Coburn

Department of Surgery, University of Toronto,

Toronto, ON, Canada
(FNRs) were analyzed. Analysis was performed based on the FNR.

Results Twenty-six articles met our inclusion criteria. SLN detection using the dye method (DM) was reviewed in 18 studies, the radiocolloid method (RM) was used in 12 studies, and both dye and radiocolloid methods (DUAL) were used in 5 studies. The DM had an overall calculated FNR of $34.7 \%$ (95\% confidence interval [CI] 21.2, 48.1). The RM had an overall calculated FNR of $18.5 \%(95 \%$ CI 9.1, 28.0). DUAL had an overall calculated FNR of $13.1 \%$ (95\% CI -0.9, 27.2).

Conclusion Application of the SLN technique may be practical for early gastric cancer. The use of DUAL for identifying SLN may yield a lower FNR than either method alone, although statistical significance was not met.

Keywords Sentinel lymph node $\cdot$ Stage migration · AJCC/UICC $\cdot$ Survival $\cdot$ Staging

\section{Introduction}

The accurate determination of lymph node (LN) status is a vital factor to determine the clinical outcomes of patients with a wide variety of tumors. Gastric cancer is no exception. With the institution of screening programs leading to the detection of gastric cancer at early stages, more patients have become eligible for less invasive resections. Gastrectomy with lymphadenectomy remains the standard of care for gastric cancer, while less invasive interventions, such as endoscopic mucosal resection (EMR), have emerged as suitable techniques for early gastric cancer (EGC). Both techniques are associated with 5-year overall survival rates greater than 90\% [1, 2]. Ongoing accumulation of pathological data has suggested 
that, for EGC, the majority of lymph nodes examined do not contain metastases [3, 4]. As extensive lymphadenectomies are not without complications, which can include bleeding, pancreatitis, subdiaphragmatic abscess, lymphorrhea, and chylous ascites [5], the rationale for performing extensive lymphadenectomies for EGC has been questioned. Therefore, limited gastric resections without an extended lymphadenectomy may be applicable to selected patients with EGC [6]. The challenge is accomplishing accurate assessment of LN status, while limiting the possible complications from a more extensive $\mathrm{LN}$ dissection. In breast cancer and melanoma, this issue has been addressed through identification of the sentinel LN (SLN). As the affected organ has predictable draining basins, the SLN is defined as the first LN to receive drainage by a primary tumor. Lymphatic mapping identifies the node most at risk for involvement, allowing for a more limited dissection and more thorough pathological examination of these sentinel nodes. This mapping technique has reduced perioperative morbidity while improving the accuracy of staging for other cancers [7]. Thus, the utility of applying the SLN mapping technique has been examined in efforts to select patients in whom no $\mathrm{LN}$ dissection or a more limited LN dissection is preferable.

Application of the SLN identification technique to EGC has several issues. First, the anatomy of the lymphatic drainage of the stomach is complex and unpredictable, facilitating the presence of skip metastases [6]. Second, the interpretation of the results and the determination of the appropriate extent of $\mathrm{LN}$ dissection are not standard in cases where the SLNs contain metastasis or micrometastases [8]. Finally, there is debate regarding the specificities of the technique. Three methods have been used for SLN mapping in gastric cancer: a dye method (DM), a radiocolloid method (RM), and a combination of the DM and RM (DUAL). For all individual techniques, which dye or radiocolloid to use and the volume to inject, as well as the location, timing, mode, and location of the injection have been examined.

To date, the diagnostic reliability and accuracy of SLN mapping in gastric cancers remains unclear. Many authors from Asia have reported accuracies of more than $96 \%$ [1, 6, 9], particularly with EGC. However, several series from Western countries have reported an SLN mapping accuracy of $80 \%$, with a false-negative rate (FNR) ranging from 15 to $20 \%$ [7, 10-12]. As gastric cancer is not as common in Western countries, it typically presents in an advanced stage. This suggests that sensitivity, specificity, accuracy, and FNR may be influenced by the T stage of the cancer. Thus, the objective of this review was to identify and synthesize findings from the literature regarding techniques of SLN biopsy in gastric cancer, and its performance as a predictor of involvement in the regional nodal basin.

\section{Methods}

Data sources

Electronic literature searches were conducted in Medline and EMBASE from January 1, 1998, to December 31, 2009, according to the search algorithm presented in Appendix A of the electronic supplementary material (ESM). Search terms included: exp Stomach Cancer/or [((gastric or stomach) adj1 cancer\$) or ((gastric or stomach) adj1 carcinoma) or ((gastric or stomach) adj1 adenocarcinoma) or ((gastric or stomach) adj1 neoplasm\$)).mp.] and [((negative or resection) adj2 margin\$).mp. or exp frozen section/or exp GASTRECTOMY/or ((gastric or stomach) adj2 resect\$). $\mathrm{mp} .[\mathrm{mp}=$ title, abstract, subject headings, heading word, drug trade name, original title, device manufacturer, drug manufacturer name] or omentectom\$.mp. or multivisceral resection\$.mp.] and [sentinel lymph node.mp] and [clinical trial/or controlled clinical trial/or exp comparative study/or meta analysis/or multicenter study/or exp practice guideline/or randomized controlled trial/] not [Case Report/or review]. A separate search of the Cochrane Central Register of Controlled Trials (1998-2009) was performed using the search term "gastric cancer". No attempt was made to locate unpublished material.

\section{Study selection and review process}

To be eligible, studies had to meet all the following criteria: (1) include patients with gastric or gastroesophageal junction adenocarcinoma, (2) be a prospective study, (3) investigate the performance of SLN navigation, and (4) be published in English in a peer-reviewed journal. Studies were excluded according to the following exclusion criteria: (1) reviews, meta-analyses, systematic reviews, abstracts, editorials or letters, case reports, and guidelines; or (2) studies involving fewer than 30 patients. If multiple studies were published from the same set of patients, the most inclusive one was included. All electronic search titles, selected abstracts, and full-text articles were independently reviewed by a minimum of two reviewers ( $\mathrm{NC}, \mathrm{RC}, \mathrm{LH}$, and $\mathrm{AB}$ ). Reference lists from review papers and relevant articles were also examined for additional studies that met our inclusion criteria. Disagreements on study inclusion/exclusion were resolved with a consensus meeting of the reviewers.

\section{Data extraction}

A systematic approach to data extraction was used to produce a descriptive summary of participants, interventions, and study findings. The following data points were collected during the review: study characteristics (country and design), patient characteristics (gender, age, tumor location, and 
stage), techniques, and outcomes (detection rate, accuracy, sensitivity, specificity, and FNR) (Tables 1, 2). The first reviewers $(\mathrm{AB}, \mathrm{MD})$ independently extracted the data and a second reviewer (RC) checked the data extraction. No attempt was made to contact authors for additional information.

\section{Data analysis}

Significant variations in the calculation of detection rate, accuracy, sensitivity, specificity, and FNR were noted in the original manuscripts. Therefore, these values were re-calculated from the original numbers reported in each publication when possible. The detection rate was defined as (total patients with identifiable SLN)/(total patients). Accuracy was defined as (number of true positives + number of true negatives)/(total patients with identifiable SLN). Sensitivity was calculated as (number of true positives)/(number of true positives + number of false negatives). Specificity was calculated as (number of true negatives/(number of false positives + number of true negatives). FNR was calculated using the definition outlined by McMasters et al. [13]: (number of false negatives)/(number of true positives). We felt that the FNR was the most important factor when considering the applicability of SLN identification, because a false-negative result (when an SLN was free of tumor cells but other nonSLNs revealed metastases) could lead to an incorrect decision about treatment [13, 14].

Statistical analyses were conducted using the R 2.10.1 software package (http://www.r-project.org, University of Auckland, Auckland, New Zealand). The FNR and 95\% confidence interval (CI) were calculated for the DM, RM, and DUAL. When studies used different techniques in the same patients, an average FNR was calculated. When studies compared different techniques in different subgroups, the data were combined for an overall FNR. We assessed the heterogeneity between studies by using the $I^{2}$ test, which indicates the presence of heterogeneity when $I^{2}$ is $\geq 50 \%$. Considering the diversity within the studies reviewed (type of dye or colloid, method of injection, location/depth of injection, $\mathrm{T}$ stage of cancer), several factors in addition to chance were anticipated to explain the differences in the FNR, so we selected the random effects model for analysis [15, 16]. Studies were weighted according to the inverse variance method. Non-overlapping $95 \%$ CIs were used to determine a statistically significant difference between groups.

\section{Results}

Search results

A total of 3608 titles were identified from the electronic and hand searches for preliminary review. After removal of duplicates and screening for relevant titles and abstracts, 58 articles were submitted for a full review. Twenty-six articles on SLN identification which satisfied the inclusion and exclusion criteria were included in this review (Fig. 1). Of the 26 studies, 24 were from Asia and 2 from Europe. A total of 1945 patients underwent surgery for gastric cancer where one SLN mapping technique or a combination of techniques and subsequent biopsy were performed.

Study and patient characteristics

All studies were prospective. Tumor stage was described in 25 studies. Twenty-one studies had exclusion criteria based on preoperative T stage. Nineteen studies excluded cT3-4, while 6 studies excluded cT2-4. Of the studies that reported T stages, 13 studies included pT3 cases in their analysis.

SLN and non-SLN metastases were identified by hematoxylin and eosin (H\&E) staining in all studies, immunohistochemistry (IHC) in 15 studies, and reverse transcriptase polymerase chain reaction (RT-PCR) in 3 studies. One study compared all three histological techniques in the same group of patients [6]. If no tumor cells were found by H\&E staining in SLNs, sections of the same node were examined by IHC for further analysis in 5 studies [5, 11, 14, 17, 18].

\section{Study findings}

The study findings are summarized in Tables 1 and 2, and Appendix B of the ESM. The outcome measures of interest included the FNR, accuracy, sensitivity, specificity, positive predictive value (PPV), and negative predictive value (NPV) for the DM, RM, and DUAL techniques in SLN identification. Figures 2, 3 and 4 depict the forest plots for studies that employed a DM, RM, and DUAL method.

\section{Results by SLN identification method}

Eighteen studies used a DM to identify the SLN [1, 3, 11, 14, 17, 19-31]. A total of 1173 patients underwent SLN identification by this method (with a range of 19-211 patients per study). Five studies used $1 \mathrm{~mL}$ of isosulfan blue [21, 22, 25-27]. Nine studies used $0.8-4 \mathrm{~mL}$ of patent blue $[1,3,11,14,17,19,23,28,30]$. Four studies used 2-5 mL of indocyanine green (ICG) [19, 24, 29, 31]. In eighteen studies, dye was endoscopically injected into the submucosa at 4 points adjacent to the tumor at laparotomy [1, 3, 11, 14, 17, 19-31]. SLNs were identified intraoperatively by direct visualisation of stained LNs. Two studies examined subserosal injection of dye at laparotomy [19, 25]. Nine studies used H\&E only [1, 3, 19, 21, 22, 25, 26, 30, 31], 8 studies used H\&E and IHC [11, 14, 17, 23, 24, 27-29], while 1 study used H\&E, IHC, and RT-PCR [20]. 
Table 1 Study and patient characteristics

\begin{tabular}{|c|c|c|c|c|}
\hline Author & Country & $N$ & Patient characteristics & Tumor characteristics \\
\hline Ajisaka [20] & Japan & 35 & NR & NR \\
\hline Arigami [6] & Japan & 61 & $\begin{array}{l}\text { Age: } 65 \text { (41-84) years } \\
\text { Male: } 44 \text { female: } 17\end{array}$ & $\begin{array}{l}\text { T1: } 53(87 \%) \\
\text { T2: } 8(13 \%) \\
\text { N0: } 61(100 \%)\end{array}$ \\
\hline Gretschel [14] & Germany & $\begin{array}{c}34(\mathrm{R}-\mathrm{SLN}=15 \\
\mathrm{D}-\mathrm{SLN}=19 \\
\mathrm{MM}=34)\end{array}$ & $\begin{array}{l}\text { Age: NR } \\
\text { Male: } 19 \text { female: } 15\end{array}$ & $\begin{array}{l}\text { T1: } 11(32 \%) \\
\text { T2: } 15(44 \%) \\
\text { T3: } 8(24 \%)\end{array}$ \\
\hline Gretschel [17] & Germany & 35 & $\begin{array}{l}\text { Age: } 60(35-84) \text { years } \\
\text { Male: } 19 \text { female: } 16\end{array}$ & $\begin{array}{l}\text { T1: } 14(40 \%) \\
\text { T2: } 18(51 \%) \\
\text { pT3: } 3(9 \%)\end{array}$ \\
\hline Hayashi [1] & Japan & 31 & $\begin{array}{l}\text { Age: } 58(42-77) \\
\text { Male: } 25 \text { female: } 6\end{array}$ & $\begin{array}{l}\text { T1: } 26(84 \%) \\
\text { T2: } 4(13 \%) \\
\text { T3: } 1(3 \%)\end{array}$ \\
\hline Hiratsuka [19] & Japan & 74 & $\begin{array}{l}\text { Age: NR } \\
\text { Male: NR female: NR }\end{array}$ & $\begin{array}{l}\mathrm{T} 1: 44(59 \%) \\
\mathrm{T} 2: 30(41 \%)^{*}\end{array}$ \\
\hline Ishizaki [26] & Japan & 101 & $\begin{array}{l}\text { Age: } 62(37-82) \text { years } \\
\text { Male: } 57 \text { female: } 44\end{array}$ & $\begin{array}{l}\text { T1: } 81(80 \%) \\
\text { T2: } 17(17 \%) \\
\text { T3: } 3(3 \%)\end{array}$ \\
\hline Isozaki [22] & Japan & 144 & $\begin{array}{l}\text { Age: } 20-80 \text { years } \\
\text { Male: NR female: NR }\end{array}$ & $\begin{array}{l}\text { T1: } 125(90 \%) \\
\text { T2: } 13(9 \%) \\
\text { T3: } 2(1 \%)\end{array}$ \\
\hline Karube [23] & Japan & 41 & $\begin{array}{l}\text { Age: } 59 \pm 10 \text { years } \\
\text { Male: } 31 \text { female: } 10\end{array}$ & $\begin{array}{l}\mathrm{T} 1: 32(78 \%) \\
\mathrm{T} 2: 7(17 \%) \\
\mathrm{T} 3: 2(5 \%)\end{array}$ \\
\hline Kim [5] & South Korea & 46 & $\begin{array}{l}\text { Age: } 53.2 \pm 12.1 \text { years } \\
\text { Male: NR female: NR }\end{array}$ & $\begin{aligned} \text { T1:32 }(70 \%) \text { T2: } 11(24 \%) \\
\text { T3:3 (6\%) N0: } 32(70 \%) \\
\text { N1: } 11(24 \%) \\
\text { N2: } 3(6 \%)\end{aligned}$ \\
\hline Kitagawa [9] & Japan & 145 & $\begin{array}{l}\text { Age: } 57 \pm 11 \text { years } \\
\text { Male: } 107 \text { female: } 38\end{array}$ & $\begin{array}{l}\text { T1: } 127(87.6 \%) \\
\text { T2: } 18(12.4 \%)\end{array}$ \\
\hline Lee [25] & South Korea & $\mathrm{SS}=71$ & $\begin{array}{l}\text { Age: } 53.9 \pm 10.8 \text { years } \\
\text { Male: } 40 \text { female: } 31\end{array}$ & $\begin{array}{l}\text { T1: } 52(73 \%) \\
\text { T2: } 19(27 \%)\end{array}$ \\
\hline & & $\mathrm{SM}=50$ & $\begin{array}{l}\text { Age: } 57.1 \pm 10.3 \text { years } \\
\text { Male: } 43 \text { female: } 7\end{array}$ & $\begin{array}{l}\mathrm{T} 1: 41(82 \%) \\
\mathrm{T} 2: 9(18 \%)\end{array}$ \\
\hline Lee [27] & South Korea & 64 & $\begin{array}{l}\text { Age: } 60 \pm 10.1 \text { years } \\
\text { Male: } 52 \text { female: } 12\end{array}$ & $\begin{array}{l}\text { T1: } 53(82.8 \%) \\
\text { T2: } 10(15.6 \%) \\
\text { T3: } 1(1.6 \%)\end{array}$ \\
\hline Lee [35] & South Korea & $\mathrm{G} 1=42$ & $\begin{array}{l}\text { Age: } 58(30-81) \text { years } \\
\text { Male: } 26 \text { female: } 16\end{array}$ & $\begin{array}{l}\text { T1: } 35(83 \%) \\
\text { T2: } 5(12 \%) \\
\text { T3: } 2(5 \%)\end{array}$ \\
\hline & & $\mathrm{G} 2=50$ & $\begin{array}{l}\text { Age: } 60(35-85) \text { years } \\
\text { Male: } 32 \text { female: } 18\end{array}$ & $\begin{array}{l}\text { T1: } 39(78 \%) \\
\text { T2: } 8(16 \%) \\
\text { T3: } 3(6 \%)\end{array}$ \\
\hline Miwa [3] & Japan & 211 & $\begin{array}{l}\text { Age: } 62(26-85) \text { years } \\
\text { Male: } 136 \text { female: } 67\end{array}$ & $\begin{array}{l}\text { T1: } 161(79.3 \%) \\
\text { T2: } 35(17.2 \%) \\
\text { T3: } 7(3.4 \%)\end{array}$ \\
\hline Miyake [28] & Japan & 76 & $\begin{array}{l}\text { Age: } 64 \text { years } \\
\text { Male: } 83 \text { female: } 37\end{array}$ & $\begin{array}{l}\text { T1a: } 70(58.3 \%)^{* *} \\
\text { T1b: } 50(41.7 \%)^{* *}\end{array}$ \\
\hline
\end{tabular}


Table 1 continued

$N R$ not reported, $N$ number of patients, $G 1$ group 1, G2 group 2, $R$-SLN radiocolloid SLN detection method, $D-S L N$ dye SLN detection method, $M M$ Maruyama index computer method, $S S$ subserosal dye injection, $S M$ submucosal dye injection

* SLN could not be identified in one patient with $\mathrm{T} 2$ gastric cancer

** Data based on 120 patients, however only 76 patients had blue dye injected

\begin{tabular}{|c|c|c|c|c|}
\hline Author & Country & $N$ & Patient characteristics & Tumor characteristics \\
\hline Mochiki [33] & Japan & 59 & $\begin{array}{l}\text { Age: } 60 \text { (36-78) years } \\
\text { Male: } 41 \text { female: } 18\end{array}$ & $\begin{array}{l}\text { T1: } 28(47.4 \%) \\
\text { T2: } 19(32.2 \%) \\
\text { T3: } 12(20.4 \%)\end{array}$ \\
\hline Nimura [24] & Japan & 84 & $\begin{array}{l}\text { Age: NR } \\
\text { Male: NR female: NR }\end{array}$ & $\begin{array}{l}\text { T1: } 75(89 \%) \\
\text { T2: } 9(11 \%)\end{array}$ \\
\hline Park [29] & South Korea & 100 & $\begin{array}{l}\text { Age: } 55.6(27-78) \text { years } \\
\text { Male: } 69 \text { female: } 31\end{array}$ & $\begin{array}{l}\text { T1a: } 49(49 \%) \\
\text { T1b: } 31(31 \%) \\
\text { T2a: } 12(12 \%) \\
\text { T2b: } 8(8 \%)\end{array}$ \\
\hline Rabin [11] & Israel & 43 & $\begin{array}{l}\text { Age: } 68.5 \text { (33-88) years } \\
\text { Male: } 26 \text { female: } 17\end{array}$ & NR \\
\hline Rino [30] & Japan & 38 & $\begin{array}{l}\text { Age: } 66.2 \pm 10(47-79) \text { years } \\
\text { Male: } 29 \text { female: } 9\end{array}$ & $\begin{array}{l}\text { T1a: } 20(52.6 \%) \\
\text { T1b: } 15(39.5 \%) \\
\text { T2a: } 1(2.6 \%) \\
\text { T2b: } 2(5.3 \%)\end{array}$ \\
\hline Ryu [21] & South Korea & 71 & $\begin{array}{l}\text { Age: } 53.9 \pm 10.8 \text { years } \\
\text { Male: } 40 \text { female: } 31\end{array}$ & $\begin{array}{l}\text { T1: } 52(73 \%) \\
\text { T2: } 19(27 \%)\end{array}$ \\
\hline Tajima [31] & Japan & 56 & $\begin{array}{l}\text { Age: } 68.4 \pm 10.1 \text { years } \\
\text { Male: } 30 \text { female: } 26\end{array}$ & $\begin{array}{l}\text { T1: } 32(57 \%) \\
\text { T2: } 16(28.5 \%) \\
\text { T3: } 8(14.5 \%)\end{array}$ \\
\hline Uenosono [32] & Japan & 104 & $\begin{array}{l}\text { Age: } 64.3(31-85) \text { years } \\
\text { Male: } 81 \text { female: } 23\end{array}$ & $\begin{array}{l}\text { T1: } 81(78 \%) \\
\text { T2: } 23(22 \%)\end{array}$ \\
\hline Yanagita [34] & Japan & 133 & $\begin{array}{l}\text { Age: NR } \\
\text { Male: NR female: NR }\end{array}$ & $\begin{array}{l}\text { T1: } 104(78 \%) \\
\text { T2: } 29(22 \%)\end{array}$ \\
\hline Zulfikaroglu [18] & Turkey & 32 & $\begin{array}{l}\text { Age: } 18 \text { (SD 13) years } \\
\text { Male: } 26 \text { female: } 6\end{array}$ & $\begin{array}{l}\text { T1: } 7(22 \%) \\
\text { T2: } 23(72 \%) \\
\text { T3: } 2(6 \%)\end{array}$ \\
\hline
\end{tabular}

all studies, the radiocolloid was endoscopically injected into the submucosa at 4 points adjacent to the tumor 16-18 $\mathrm{h}$ preoperatively. SLNs were detected intraoperatively by utilizing a handheld gamma probe. LNs that absorbed 10 times more radioisotope than background levels or more than $10 \%$ of the value of the node with the highest radioisotope in each patient were classified as SLNs. Three studies used H\&E only [1, 9, 33], 8 studies used H\&E and IHC [5, 14, 17, 18, 23, 27, 32, 34], while 1 study used H\&E, IHC, and RT-PCR, and reported the performance of each histological technique separately in the same group of patients [6]. The detection rate for the RM ranged from 84.4 to $98.5 \%$. Calculated accuracies ranged from 71.4 to $100 \%$, calculated sensitivities ranged from 50.0 to $100 \%$, calculated specificities were $100 \%$, and the calculated FNR ranged from 0 to $100 \%$. The overall calculated FNR for the RM was $18.5 \%$ (95\% CI 9.1, 28.0). For the studies utilizing ${ }^{99 \mathrm{~m}} \mathrm{Tc}$-tin colloid, the FNR was $23.9 \%$ (95\% CI 10.0, 37.8) (forest plot not shown). For the studies utilizing ${ }^{99 \mathrm{~m}} \mathrm{Tc}$-sulfur colloid, the FNR was $11.4 \%$ (95\% CI -1.7, 24.6) (forest plot not shown). 
Table 2 Sentinel lymph node identification by the three methods

\begin{tabular}{lllll}
\hline Author & Method & FNR \\
$(\%)$ & Accuracy $(\%)$ & Sensitivity $(\%) \quad$ Specificity $(\%)$ & PPV $(\%) \quad$ NPV $(\%)$
\end{tabular}

\begin{tabular}{|c|c|c|c|c|c|c|c|c|}
\hline \multicolumn{9}{|l|}{ (a) Dye method } \\
\hline Hiratsuka [19] & ICG & 73 & 11.1 & 98.6 & 90 & 100 & 100 & 98.4 \\
\hline \multirow[t]{2}{*}{ Ajiska [20] } & $\mathrm{PB}$ & 35 & 40 & 94.3 & 71.4 & 100 & 100 & 93.3 \\
\hline & PB-RT-PCR & 35 & 16.7 & 97.1 & 83.7 & 100 & 100 & 96.6 \\
\hline Hayashi [1] & $\mathrm{PB}$ & 28 & 16.7 & 96.4 & 85.7 & 100 & 100 & 95.5 \\
\hline Miwa [3] & PB & 195 & 12.9 & 97.9 & 88.6 & 100 & 100 & 97.6 \\
\hline Ryu [21] & IB & 65 & 63.6 & 89.2 & 61.1 & 100 & 100 & 87 \\
\hline Gretschel [14] & PB & 19 & 0 & 100 & 100 & 100 & 100 & 100 \\
\hline Isozaki [22] & IB & 99 & 53.3 & 91.9 & 65.2 & 100 & 100 & 90.5 \\
\hline Karube [23] & PB & 36 & 30 & 91.7 & 76.9 & 100 & 100 & 88.5 \\
\hline \multirow[t]{2}{*}{ Nimura [24] } & ICG & 84 & 57.1 & 95.2 & 63.6 & 100 & 100 & 94.8 \\
\hline & ICG w/IREE & 84 & 0 & 100 & 100 & 100 & 100 & 100 \\
\hline \multirow[t]{2}{*}{ Lee [25] } & $\mathrm{IB} ; \mathrm{SS}$ & 65 & 63.6 & 89.2 & 61.1 & 100 & 100 & 87 \\
\hline & IB; SM & 47 & 120 & 87.2 & 45.5 & 100 & 100 & 85.7 \\
\hline Ishizaki [26] & IB & 101 & 16.7 & 97 & 85.7 & 100 & 100 & 96.4 \\
\hline Lee [27] & IB & 52 & 66.7 & 88.5 & 60 & 100 & 100 & 86 \\
\hline Miyake [28] & PB & 76 & 36.4 & 94.7 & 73.3 & 100 & 100 & 93.8 \\
\hline \multirow[t]{6}{*}{ Park [29] } & ICG; H\&E; total & 94 & 27.3 & 96.8 & 78.6 & 100 & 100 & 96.4 \\
\hline & ICG; H\&E; T1 & 74 & 50 & 97.3 & 66.7 & 100 & 100 & 97.1 \\
\hline & ICG; H\&E; T2 & 20 & 14.3 & 95 & 87.5 & 100 & 100 & 92.3 \\
\hline & ICG; IHC; total & 45 & 40 & 91.1 & 71.4 & 100 & 100 & 88.6 \\
\hline & ICG; IHC; T1 & 38 & 60 & 92.1 & 62.5 & 100 & 100 & 90.9 \\
\hline & ICG; IHC; T2 & 7 & 20 & 85.7 & 83.3 & 100 & 100 & 50 \\
\hline Rabin [11] & $\mathrm{PB}$ & 33 & 15 & 90.9 & 87 & 100 & 100 & 76.9 \\
\hline Rino [30] & PB & 35 & 0 & 100 & 100 & 100 & 100 & 100 \\
\hline Gretschel [17] & PB & 34 & 50 & 76.5 & 66.7 & 100 & 100 & 55.6 \\
\hline \multirow[t]{5}{*}{ Tajima [31] } & ICG; total & 54 & 54.5 & 88.9 & 64.7 & 100 & 100 & 86 \\
\hline & ICG; T1 & 36 & 16.7 & 97.2 & 85.7 & 100 & 100 & 96.7 \\
\hline & ICG; T2-3 & 18 & 100 & 72.2 & 50 & 100 & 100 & 61.5 \\
\hline & ICG; Preop & 31 & 0 & 100 & 100 & 100 & 100 & 100 \\
\hline & ICG; Intraop & 23 & 150 & 73.9 & 40 & 100 & 100 & 68.4 \\
\hline \multicolumn{9}{|c|}{ (b) Radiocolloid method } \\
\hline Kitagawa [9] & Tin & 138 & 9.1 & 98.6 & 91.7 & 100 & 100 & 98.3 \\
\hline Hayashi [1] & Tin & 28 & 40.0 & 92.9 & 71.4 & 100 & 100 & 91.3 \\
\hline Gretschel [14] & Sulfur & 14 & 12.5 & 92.9 & 88.9 & 100 & 100 & 83.3 \\
\hline Karube [23] & Tin & 37 & 33.3 & 91.9 & 75 & 100 & 100 & 89.3 \\
\hline Kim [5] & Tin & 43 & 18.2 & 95.3 & 84.6 & 100 & 100 & 93.8 \\
\hline \multirow[t]{3}{*}{ Uenosono [32] } & Tin; total & 99 & 31.6 & 93.9 & 76 & 100 & 100 & 92.5 \\
\hline & Tin; T1 & 78 & 0.0 & 100.0 & 100 & 100 & 100 & 100 \\
\hline & Tin; T2 & 21 & 50.0 & 71.4 & 66.7 & 100 & 100 & 33.3 \\
\hline Zulfikaroglu [18] & Sulfur & 31 & 0.0 & 100.0 & 100 & 100 & 100 & 100 \\
\hline \multirow[t]{3}{*}{ Arigami [6] } & Tin; H\&E & 61 & 100.0 & 91.8 & 50 & 100 & 100 & 91.1 \\
\hline & Tin; IHC & 61 & 62.5 & 91.8 & 61.5 & 100 & 100 & 90.6 \\
\hline & Tin; RT-PCR & 61 & 4.5 & 98.4 & 95.7 & 100 & 100 & 97.4 \\
\hline Lee [27] & Tin & 45 & 55.6 & 88.9 & 64.3 & 100 & 100 & 86.1 \\
\hline
\end{tabular}


Table 2 continued

\begin{tabular}{|c|c|c|c|c|c|c|c|c|}
\hline Author & Method & $N$ & $\begin{array}{l}\text { FNR } \\
(\%)\end{array}$ & Accuracy $(\%)$ & Sensitivity (\%) & Specificity (\%) & PPV (\%) & NPV $(\%)$ \\
\hline \multirow[t]{4}{*}{ Mochiki [33] } & Sulfur; T1 & 28 & 0.0 & 100.0 & 100 & 100 & 100 & 100 \\
\hline & Sulfur; T2 & 19 & 9.1 & 94.7 & 91.7 & 100 & 100 & 87.5 \\
\hline & Sulfur; T3 & 12 & 60.0 & 75.0 & 62.5 & 100 & 100 & 57.1 \\
\hline & Sulfur; total & 59 & 20.0 & 93.2 & 83.3 & 100 & 100 & 89.7 \\
\hline Gretschel [17] & Sulfur & 34 & 9.1 & 94.1 & 91.7 & 100 & 100 & 83.3 \\
\hline Yanagita [34] & Tin & 131 & 0.0 & 100.0 & 100 & 100 & 100 & 100 \\
\hline \multicolumn{9}{|l|}{ (c) Dual method } \\
\hline Hayashi [1] & Tin + PB & 31 & 0.0 & 100.0 & 100 & 100 & 100 & 0 \\
\hline Karube [23] & Tin + PB & 41 & 8.3 & 97.6 & 92.3 & 100 & 100 & 96.6 \\
\hline Lee [27] & Tin + IB & 50 & 41.7 & 90.0 & 70.6 & 100 & 100 & 86.8 \\
\hline Gretschel [17] & Sulfur + PB & 34 & 9.1 & 94.1 & 91.7 & 100 & 100 & 83.3 \\
\hline \multirow[t]{2}{*}{ Lee [35] } & Tin + ICG (pick-up) & 23 & 50.0 & 91.3 & 66.7 & 100 & 100 & 89.5 \\
\hline & Tin + ICG (Basin) & 48 & 16.7 & 95.8 & 85.7 & 100 & 100 & 94.4 \\
\hline
\end{tabular}

$F N R$ false-negative rate, $P P V$ positive predictive value, $N P V$ negative predictive value, $I C G$ indocyanine green, $P B$ patent blue, $R T-P C R$ reverse transcriptase polymerase chain reaction, $I B$ isosulfan blue, IREE infrared ray electronic endoscopy, $S S$ subserosal, $S M$ submucosal, $H \& E$ hematoxylin \& eosin, IHC immunohistochemistry, Preop preoperative administration of contrast, Intraop intraoperative administration of contrast, Tin ${ }^{99 \mathrm{~m}}$ Tc-Tin colloid, Sulfur ${ }^{99 \mathrm{~m}}$ Tc-sulfur colloid, Pick-up pick-up SLN biopsy method, Basin basin dissection method

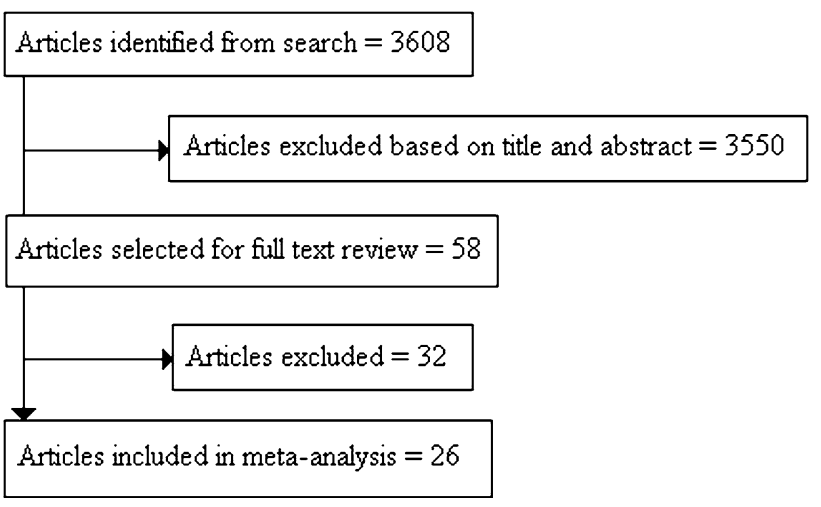

Fig. 1 Article selection flow

Five studies $[1,17,23,27,35]$ used DUAL to identify the SLN. A total of 298 patients underwent SLN identification by this method (with a range of $31-92$ patients per study). Three studies used $1-1.2 \mathrm{~mL}$ of patent blue [1, 17, 23]; one study used $1 \mathrm{~mL}$ of isosulfan blue [27]; and one study used $2 \mathrm{~mL}$ of ICG [35]. Four studies used $2 \mathrm{~mL}$ of ${ }^{99 \mathrm{~m}}$ Tc-tin colloid $[1,23,27,35]$ and one study used $0.4 \mathrm{~mL}$ of ${ }^{99 \mathrm{~m}} \mathrm{Tc}$-sulfur colloid [17]. In all studies, the radiocolloid was endoscopically injected into the submucosa at 4 points adjacent to the tumor 16-18 $\mathrm{h}$ preoperatively, and the dye was endoscopically injected into the submucosa at 4 points adjacent to the tumor at laparotomy. One study used $\mathrm{H} \& \mathrm{E}$ only [1], and 4 studies used H\&E and IHC [17, 23, 27, 35]. The detection rate for DUAL ranged from 54.8 to $100 \%$. Calculated accuracies ranged from 90.0 to $100 \%$, calculated sensitivities ranged from 66.7 to $100 \%$, calculated specificities were $100 \%$, and the calculated FNR ranged from 0 to $50.0 \%$. The overall calculated FNR for DUAL was $13.1 \%$ (95\% CI -0.9, 27.2).

Four studies reported results by $\mathrm{T}$ stage [29, 31-33], representing 351 patients. Overall FNR was calculated for T1 tumors and T2-3 tumors to compare the performance of SLN identification between these stages. The FNR for T1 tumors was $22.5 \%$ (95\% CI -7.8, 52.9) (forest plot not shown) and for T2-3 tumors, it was 31.1\% (95\% CI 11.7, 50.4) (forest plot not shown).

\section{Discussion}

The current accepted standard of care for resection of gastric cancer with curative intent is gastrectomy with D2 lymphadenectomy. Over time, the question has arisen as to whether performing an extensive lymphadenectomy is necessary for EGC, as less extensive resections have been shown to have equivalent survival rates. However, less extensive resection requires accurate staging, and thus, though the SLN makes good conceptual sense, it must also have a clinically acceptable FNR. Our examination of the literature shows that there were several variations in the technique applied to the identification of SLNs in gastric cancer: a DM (using blue dye or ICG), an RM (using tin or sulfur colloid), and DUAL. Statistically significant differences between these techniques were not identified in our analysis. Only four studies [29, 31-33] reported results by 
Fig. 2 False negative rate for the dye method. FNR false negative rate, $C I$ confidence interval
Fig. 3 False negative rate for the radiocolloid method. FNR false negative rate, $C I$ confidence interval

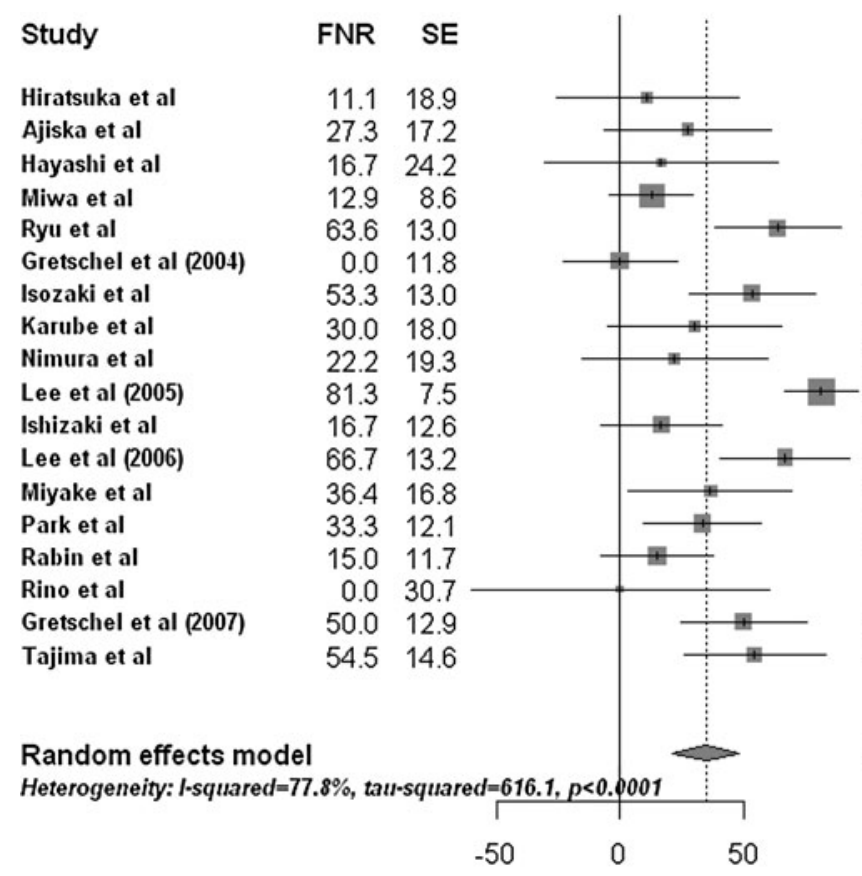

$95 \%-\mathrm{Cl}$

Weight

$11.10 \quad[-25.94 ; 48.14]$

$27.30 \quad[-6.41 ; 61.01]$

$16.70 \quad[-30.73 ; 64.13]$

$12.90 \quad[-3.96 ; 29.76]$

$63.60 \quad[38.12 ; 89.08]$

$0.00 \quad[-23.13 ; 23.13]$

$53.30 \quad[27.82 ; 78.78]$

$30.00 \quad[-5.28 ; 65.28]$

$22.20 \quad[-15.63 ; 60.03]$

81.30 [66.60; 96.00]

$16.70 \quad[-8.00 ; 41.40]$

66.70 [40.83:92.57]

$36.40 \quad$ [ $3.47 ; 69.33]$

$33.30 \quad[9.58 ; 57.02]$

$15.00 \quad[-7.93 ; 37.93]$

$0.00 \quad[-60.17 ; 60.17]$

$50.00 \quad[24.72 ; 75.28]$

$54.50 \quad[25.88 ; 83.12]$

$4.8 \%$

$5.2 \%$

$3.9 \%$

$6.8 \%$

$6.0 \%$

$6.2 \%$

$6.0 \%$

$5.0 \%$

$4.8 \%$

$7.0 \%$

$6.1 \%$

$5.9 \%$

$5.2 \%$

$6.2 \%$

$6.2 \%$

$3.0 \%$

$6.0 \%$

$5.7 \%$

$34.68[21.24 ; 48.12] \quad 100 \%$

$\begin{array}{lrr}\text { Study } & \text { FNR } & \text { SE } \\ \text { Kitagawa et al } & 9.1 & 10.2 \\ \text { Hayashi et al } & 40.0 & 23.1 \\ \text { Gretschel et al (2004) } & 12.5 & 20.5 \\ \text { Karube et al } & 33.3 & 18.7 \\ \text { Kim et al } & 18.2 & 17.1 \\ \text { Uenosono et al } & 31.6 & 12.7 \\ \text { Zulfikaroglu et al } & 0.0 & 17.2 \\ \text { Arigami et al } & 31.4 & 16.5 \\ \text { Lee et al (2006) } & 55.6 & 15.7 \\ \text { Mochiki et al } & 20.0 & 12.1 \\ \text { Gretschel et al (2007) } & 9.1 & 10.2 \\ \text { Yanagita et al } & 0.0 & 9.0\end{array}$

Random effects model

Heterogeneity: 1 -squared $=30.1 \%$, tau-square $=80.51, p=0.15 .13$
$95 \%-\mathrm{Cl}$ Weight

$9.10[-10.89 ; 29.09] 12.7 \%$ $40.00[-5.28 ; 85.28] \quad 3.8 \%$

$12.50[-27.68 ; 52.68] \quad 4.7 \%$

$33.30[-3.35 ; 69.95] \quad 5.4 \%$

$18.20[-15.32 ; 51.72] \quad 6.3 \%$

$31.60[6.71 ; 56.49] \quad 9.7 \%$

$0.00[-33.71 ; 33.71] \quad 6.2 \%$

$31.40[-0.94 ; 63.74] \quad 6.6 \%$

$55.60[24.83 ; 86.37] \quad 7.2 \%$

$20.00[-3.72 ; 43.72] \quad 10.3 \%$

$9.10[-10.89 ; 29.09] 12.7 \%$

$0.00[-17.64 ; 17.64] 14.5 \%$

18.54 [ $9.06 ; 28.02] 100 \%$

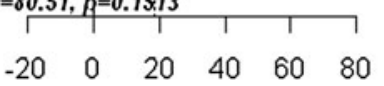

Fig. 4 False negative rate for the DUAL method (dye method and radiocolloid method). FNR false negative rate, $C I$ confidence interval

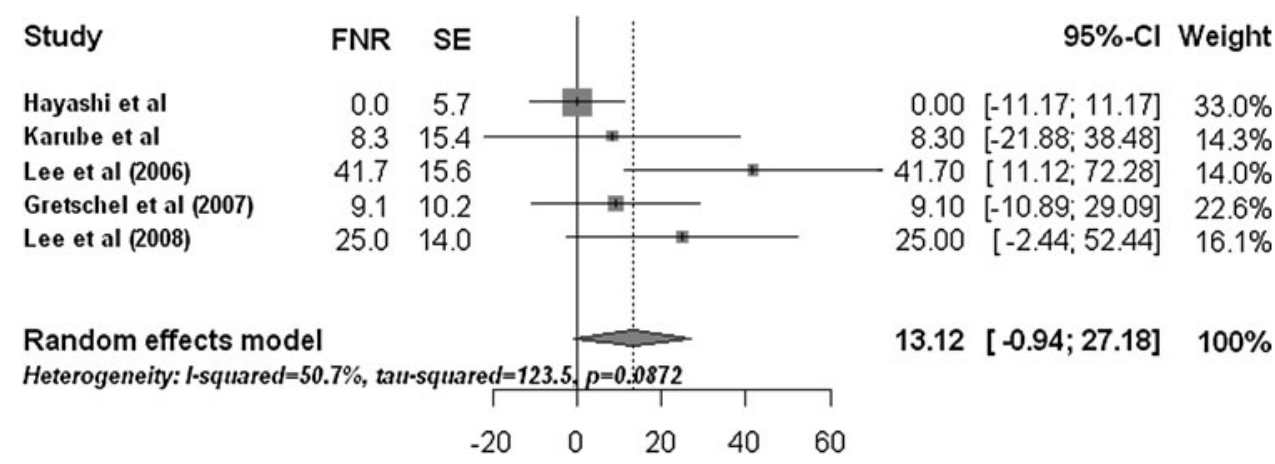


T stage, and we found no statistically significant difference between $\mathrm{T} 1$ and T2-3 in our analysis.

Implications of the false-negative rate

The FNR is the most clinically important statistic to consider when evaluating the utility of SLN identification for any type of cancer. FNR was defined in the majority of the studies as (number of false negatives)/(number of true positives + number of false negatives). When this formula was utilized by the primary authors, the range of FNRs for the DM was $0-60 \%$, with values of $0-38.5 \%$ for the RM, and $0-33.3 \%$ for DUAL (results not shown). However, a more clinically appropriate definition of FNR describes the percentage of patients with nodal metastases who would have been incorrectly designated as node-negative if the SLN biopsy had been used [13]. Thus, we re-calculated the FNR for all the manuscripts in this review, utilizing the definition outlined by McMasters et al. [13]. While this definition of FNR yields a higher FNR than those in the original manuscripts, it allows for comparison to other cancers, such as breast cancer, where SLN identification is an established technique. With the re-calculated FNR, we found a range of $0-150 \%$. This wide range, with a clinically unacceptable FNR at the high end of the range, originates from several caveats regarding the use of SLN in gastric cancer that require further discussion. The major factors affecting the FNR appeared to be: (1) skip metastases; (2) complex, multidirectional lymphatic drainage patterns; (3) injection techniques; (4) T-staging effects; and (5) the definition of micrometastases.

First, patients may be incorrectly staged as node-negative due to skip metastases, or inaccuracies in pathological examination. With a reported rate of approximately $10 \%$, skip metastases are one cause of false negatives and their presence is an important consideration in the application of the SLN identification to gastric cancer [36]. Hayashi et al. [1] reported half of the SLNs detected by a radiotracer method were stained with dye, and half of the SLNs detected by the dye method were radioactive; however, this means that half of the SLNs identified using each technique (dye or radiotracer) were classified as non-SLNs by the other identification technique. They explained this discrepancy as originating from the lack of consistency in the exact location of injection, as the radiocolloid was injected 16-18 h preoperatively. Consequently, mild inflammation of the gastric mucosa observed after injection of the tin colloid may have modified the lymphatic flow from the primary lesion [1, 23], thus reflecting differences in the accumulation of these tracers in SLNs.

Second, anatomic differences in the complexity of lymphatic drainage must be considered when comparing stomach cancer to breast cancer SLN biopsies. The lymphatic drainage of the breast is relatively straightforward: most of the lymphatic drainage will go to the axilla. The lymphatic system of the stomach, however, is much more complex, as the gastric wall is supplied with a very rich submucosal lymphatic network that freely communicates with other equally complex intermuscular and subserosal lymphatic networks [37, 38]. This rich lymphatic network, dilution and widespread distribution of the dye and radiocolloid, and multi-directional lymphatic flow should be considered in order to minimize false-negative cases [22]. Lee et al. [25] compared subserosal injection with submucosal injection of dye, followed by gastrectomy and lymphadenectomy. While they were unable to find significant differences between the two techniques in terms of FNR (reported 54.5\% with submucosal injection; reported $38.9 \%$ for subserosal injection) or sensitivity ( $45.5 \%$ vs. $61.1 \%)$, they preferred subserosal injection of dye as it was technically easier and led to shorter operative times. Other groups that examined submucosal injection of dye reported FNRs of $0-40.0 \%$ (calculated FNRs of $0-66.7 \%$ ). One study examined the difference between a pick-up method (where only stained and/or radioactive nodes were removed) and a basin dissection method (the entire LN basin was removed if stained and/or if radioactive nodes were present) [35]. The detection rate for the pick-up method was much poorer (54.8 vs. $96.0 \%)$, as were the calculated FNRs $(50.0 \%, 95 \%$ CI 6.8, 93.2, and $16.7 \%$, $95 \%$ CI $-15.1,48.4)$, suggesting that removal of entire nodal basins can significantly decrease the FNR rate.

Third, Ishizaki et al. [26] found that the time interval from injection to dissection altered the FNR and accuracy. During the initial phase of their study, they had 3 false negatives $(17.0 \%)$ with an accuracy rate of $97.0 \%$. With a longer time interval, the FNR decreased to $0 \%$ and the accuracy of SLN identification increased to $100 \%$.

Fourth, the T-stage depth of invasion may significantly change the accuracy of SLN biopsy. T1 cancers were postulated to be the most reasonable stage for the application of SLN identification in an effort to avoid a completion lymphadenectomy. One study found that the sensitivity of performing DUAL SLN identification decreased as the tumors' depth of invasion increased [27]. Another study reported FNRs of $0 \%$ for pT1 lesions, $9.1 \%$ for pT2 lesions, and $60.0 \%$ for pT3 lesions [33]. These results are consistent with other studies where data were reported according to $\mathrm{T}$ stage $[31,32]$. Some authors have proposed that the primary cancer may have destroyed the lymphatic drainage from the submucosal layer at the injection sites around advanced tumors, leading to an increase in false negatives with more advanced stages of gastric cancer [33]. Additionally, lymphatics to the positive lymph nodes may be occluded with tumor cells [19, 33]. While many authors stated that SLN identification was most accurate in T1 lesions [6, 19, 22, 29-31, 33, 34], we 
cannot substantiate this, as we found a lack of statistically significant differences in the FNR between T1 and T2-3 cancers. Unfortunately, the number of cases included in this analysis was small, possibly obscuring a true difference.

Finally, the ability of the SLN biopsy technique to identify micrometastases (metastases $<2 \mathrm{~mm}$ ) and their clinical significance in gastric cancer is another area of concern. Small metastases located in the marginal sinus of lymph nodes are generally identifiable without difficulty by $H \& E$; however, small tumor cell clusters in the medulla are harder to detect [23]. IHC emerged as a superior technique to H\&E for identifying micrometastases [28], though more expensive [33]. RT-PCR with a carcinoembryonic antigen (CEA) primer is also a superior technique to discover micrometastases. Arigami et al. [6] reported that the incidence of micrometastases identified by $\mathrm{H} \& \mathrm{E}$ was $8.2 \%$, with the incidence being $13.1 \%$ by IHC, and $36.1 \%$ for RT-PCR. In patients with cT1N0 tumors, the calculated FNR and accuracy, using RT-PCR, were $4.5 \%$ and $98.4 \%$, respectively [6]. Performing routine RT-PCR is cumbersome and unnecessary, especially for non-SLNs. IHC was considered more convenient than RT-PCR. Therefore, if SLNs are negative by $\mathrm{H} \& \mathrm{E}$, examination of other sections by IHC is reasonable [5, 11, 17, 18]. Additionally, the sectioning of LNs affects accuracy. In the majority of the studies, the SLNs were bivalved across their longest axis and sent for analysis. Increasing the number of sections used for routine staining and immunostaining has been shown to increase the detection rate [28], by decreasing sampling error.

\section{Techniques of SLN identification}

The DM was the most commonly investigated technique for SLN identification. Isosulfan blue and patent blue were used most commonly in SLN identification in EGC because of their good lymphatic affinity, and visibility in the operative field, even when the lymphatic vessels were very thin [26]. While some authors performed subserosal injection of the dye [19, 25], most authors endoscopically injected the dye submucosally at laparotomy. The latter technique would be most applicable to EGC, which is often difficult to palpate or visualize at laparotomy [3], or identify laparoscopically. Hayashi et al. [1] offer suggestions on the use of blue dyes. First, because the pericardial area or prepyloric area is rich in fat and lacking peritoneum, skeletonization of vessels in these areas may be required to detect positive staining nodes. Second, the identification and removal of SLNs must be performed expeditiously, as dye can flow into non-SLNs while the staining of the primary lymph nodes or lymph vessels diminishes in 2 or $3 \mathrm{~h}$.

ICG was initially developed to test liver and circulatory function [39], and has since been applied to SLN detection and biopsy. When ICG was injected endoscopically adjacent to a tumor, one early study found that it bound to albumin and was carried through the lymphatics more specifically than other dyes [40], suggesting a greater affinity for lymphatics [24, 35]. Nimura et al. found that their ability to detect SLNs was enhanced by using infrared ray electronic endoscopy (IREE) technology. While SLNs stained with ICG were not always visible to all attending surgeons and endoscopists, SLNs illuminated with IREE were clear enough to be identified by all personnel [24]. No clear benefit of ICG over the blue dyes could be identified based on our statistical analysis.

The colloid used most commonly for the RM was a ${ }^{99} \mathrm{~m}$ Tc-tin colloid, a larger colloid that can measure up to $1000 \mathrm{~nm}$ in size, with variation in size according to the conditions of its preparation [1, 23]. Smaller-particle radiotracers, such as ${ }^{99 \mathrm{~m}}$ Tc-sulfur colloid or ${ }^{99 \mathrm{~m}}$ Tc-colloidal albumin, are widely used in the United States and Europe, but are not commercially available in Japan [1] and other countries in Asia, where the majority of the studies reviewed originated. Several authors reported that in the preparation of the ${ }^{99 \mathrm{~m}}$ Tc-tin colloid for use in the operating room, they used a filtered syringe to reduce the size of the tin colloid. A higher FNR for the RM versus the DM technique was attributed to the large particle size of the unfiltered colloid used in one study [23]. In areas where ${ }^{99 \mathrm{~m}}$ Tc-sulfur colloid or ${ }^{99 \mathrm{~m}}$ Tc-colloidal albumin are not available, filtration of the ${ }^{99 \mathrm{~m}} \mathrm{Tc}$-tin colloid may be a reasonable maneuver. Injection of the ${ }^{99 \mathrm{~m}}$ Tc-tin colloid is associated with a mild amount of mucosal inflammation (as noted above), and the question of its affect on the lymphatic drainage of the tumor has been raised. Although the authors who used a ${ }^{99 \mathrm{~m}}$ Tc-sulfur colloid did not report any inflammation upon injection of the radiocolloid in the submucosa, no statistically significant difference between the two radiocolloids was identified in this review.

Lymphoscintigraphy was performed in several of the RM studies [1, 5, 6, 17, 27]; however, some authors found that it was unhelpful and reported detection rates of $0.0 \%$ [27], $6.7 \%$ [1], and $30.0 \%$ [5]. The low detection rates may be due to the fact that most lymph nodes with uptake on lymphoscintigraphy were too close to the main tumor to be distinguished [1, 27, 36], whereas in breast cancers and melanoma, the SLN is located further from the primary cancer. Second, the uptake of tracers in the lymph nodes versus the main tumor was reported to be lower in the stomach when compared to that in other sites [36]; consequently, as the proportion of radioisotope uptake is much lower in draining nodes than in the main tumor, lymph nodes will not show up as well on lymphoscintigraphy.

DUAL was applied in only a few studies. As such, evidence to substantiate its benefit over the RM and DM is limited, resulting in large CIs. Many of the same issues 
occurring with the DM and RM are present with DUAL. From a technical standpoint, one fundamental issue limiting the use of DUAL is the need for two separate endoscopies. In busy centers, this may place a limit on its applicability. While there was a trend towards improvement in the FNR with DUAL, no clear benefit could be established between the DM, RM, or DUAL based on our statistical analysis.

Sentinel node detection in both breast cancer and melanoma patients is superior with DUAL. In a pivotal trial, McMasters et al. [41] showed an FNR of $11.8 \%$ for singleagent detection (RM or DM) and $5.8 \%$ for DUAL in a study of 562 patients. In a recent meta-analysis of the utility of SLN identification in melanoma, Valsecchi et al. [42] reviewed over 70 studies that included more than 25000 patients, and found an FNR of $12.5 \%$ overall (95\% CI 11-14.2\%). Using the DUAL method, some authors [1, 17, 23] were able to achieve an FNR of $0-9.1 \%$ for gastric cancer, which may be an acceptable range given the data from both breast cancer and melanoma studies. Caution needs to be exercised in the adoption of DUAL for gastric cancer based on these results, because of the lack of power in the above studies, as these gastric cancer studies of DUAL represent only 227 patients (Table 2). Furthermore, five studies were performed examining the use of the DUAL in EGC, and only three of these five studies were able to achieve an FNR of less than 10\%, which brings the reproducibility of these kinds of results into question.

\section{Limitations in the application of SLN identification} to gastric cancer

There are several limitations of this review. There may be a publication bias to present only favorable results for SLN biopsy results. All of the reviewed studies were conducted in large, high-volume institutions, and thus the results may not be applicable to the general population. Additionally, significant heterogeneity was found in the analysis of the FNR, suggesting that other factors, such as $\mathrm{T}$ stage, injection technique, type of dye, timing of injection, time interval from time of injection to dissection, or pathological examination may also play a role in the variability of the FNR. Furthermore, the wide CIs obtained in our analyses are reflective of the low numbers available for analysis. A large, standardized randomized control trial comparing techniques, or application to a specific population of patients (i.e., T1 disease), would be necessary to draw further conclusions.

\section{Conclusion}

Application of SLN identification to EGC is not yet the standard of care. Many of the studies that have examined the utility of SLN identification thus far have been small prospective series and lack the power to show a superior technique. When compared to using a single agent (DM or $\mathrm{RM}$ ), DUAL appears to have a trend towards an improvement in the FNR, but the trend lacks statistical significance. There was no statistically significant impact on the FNR between blue and green dyes, nor were there statistically significant differences between ${ }^{99 \mathrm{~m}} \mathrm{~T}$-sulfur colloids and filtered ${ }^{99} \mathrm{~m}$ T-tin colloid. While most authors concluded that the application of SLN identification was safest for $\mathrm{T} 1$ disease, we found no statistically significant difference in the FNR for T1 versus T2-3 disease.

Acknowledgments This research is funded by the Canadian Cancer Society (Grant \#019325). Dr. Coburn has received the Career Scientist Award through the Ontario Ministry of Health and Long-Term Care.

\section{References}

1. Hayashi H, Ochiai T, Mori M, Karube T, Suzuki T, Gunji Y, et al. Sentinel lymph node mapping for gastric cancer using a dual procedure with dye- and gamma probe-guided techniques. J Am Coll Surg. 2003;196(6):68-74.

2. Goto O, Fujishiro M, Kodashima S, Ono S, Omata M. Outcomes of endoscopic submucosal dissection for early gastric cancer with special reference to validation for curability criteria. Endoscopy. 2009;41(2):118-22 (Epub 2009 Feb 12).

3. Miwa K, Kinami S, Taniguchi K, Fushida S, Fujimura T, Nonomura A. Mapping sentinel nodes in patients with early-stage gastric carcinoma. Br J Surg. 2003;90:178-82.

4. Gotoda T, Yanagisawa A, Sasako M, Ono H, Nakanishi Y, Shimoda $\mathrm{T}$, et al. Incidence of lymph node metastasis from early gastric cancer: estimation with a large number of cases at two large centers. Gastric Cancer. 2000;3:219-25.

5. Kim MC, Kim HH, Jung GJ, Lee JH, Choi SR, Kang DY, et al. Lymphatic mapping and sentinel node biopsy using ${ }^{99 \mathrm{~m}} \mathrm{Tc}$ tin colloid in gastric cancer. Ann Surg. 2004;239(3):383-7.

6. Arigami T, Natsugoe S, Uenosono Y, Mataki Y, Ehi K, Higashi $\mathrm{H}$, et al. Evaluation of sentinel node concept in gastric cancer based on lymph node micrometastasis determined by reverse transcription-polymerase chain reaction. Ann Surg. 2006;243: 341-7.

7. Becher RD, Shen P, Steward JH IV, Geisinger KR, McCarthy LP, Levine EA. Sentinel lymph node mapping for gastric adenocarcinoma. Am Surg. 2009;75:710-4.

8. Aikou T, Kitagawa Y, Kitajima M, Uenosono Y, Bilchik A, Martinez S, et al. Sentinel lymph node mapping with GI cancer. Cancer Metastasis Rev. 2006;25:269-77.

9. Kitagawa Y, Fujii H, Mukai M, Kubota T, Otani Y, Kitajima M. Radio-guided sentinel node detection for gastric cancer. Br J Surg. 2002;89:604-8.

10. Cozzaglio L, Bottura R, Di Rocco M, Gennari L, Doci R. Sentinel lymph node biopsy in gastric cancer: possible applications and limits. EJSO. 2011;37:55-9.

11. Rabin I, Chikman B, Halpern Z, et al. Sentinel node mapping for gastric cancer. Isr Med Assoc J. 2006;8:40-3.

12. Orsenigo E, Tomajer V, Di Palo S, Albarello L, Doglioni C, Masci E, et al. Sentinel node mapping during laparoscopic distal gastrectomy for gastric cancer. Surg Endosc. 2008;22:118-21. 
13. McMasters KM, Giuliano AE, Ross MI, Reintgen DS, Hunt KK, Byrd DR, et al. Sentinel-lymph-node biopsy for breast cancernot yet the standard of care. N Engl J Med. 1998;339(14):990-5.

14. Gretschel S, Bembenek A, Ulmer Ch, Hunerbein M, Markwardt J, Schneider U, et al. Prediction of gastric cancer lymph node status by sentinel lymph node biopsy and the Maruyama computer model. EJSO. 2005;31:393-400.

15. Hyde CJ, Stanworth SJ, Murphy MF. Can you see the wood for the trees? Making sense of forest plots in systematic reviews. Transfusion. 2008;48(2):218-20.

16. Hyde CJ, Stanworth SJ, Murphy MF. Can you see the wood for the trees? Making sense of forest plots in systematic reviews 2 . Analysis of the combined results from the included studies. Transfusion. 2008;48(4):580-3.

17. Gretschel S, Bembenek A, Hunerbein M, Dressel S, Schneider W, Schlag PM. Efficacy of different technical procedures for sentinel lymph node biopsy in gastric cancer staging. Ann Surg Oncol. 2007;14(7):2028-35.

18. Zulfikaroglu B, Koc M, Ozmen MM, Kucuk NO, Ozlap NO, Aras G. Intraoperative lymphatic mapping and sentinel lymph node biopsy using radioactive tracer in gastric cancer. Surgery. 2005;138(5):899-904.

19. Hiratsuka M, Miyashiro I, Ishikawa O, Furukawa H, Motomura $\mathrm{K}$, Ohigashi $\mathrm{H}$, et al. Application of sentinel node biopsy to gastric cancer surgery. Surgery. 2001;129:335-40.

20. Ajisaka $\mathrm{H}$, Miwa $\mathrm{K}$. Micrometastases in sentinel nodes of gastric cancer. Br J Cancer. 2003;89(4):676-80.

21. Ryu KW, Lee JH, Kim HS, Kim YW, Choi IJ, Bae JM. Prediction of lymph nodes metastasis by sentinel node biopsy in gastric cancer. EJSO. 2003;29:895-9.

22. Isozaki H, Kimura T, Tanaka N, Satoh K, Matsumoto S, Ninomiya M, et al. An assessment of the feasibility of sentinel lymph node-guided surgery for gastric cancer. Gastric Cancer. 2004;7: 149-53.

23. Karube $T$, Ochiai $T$, Shimada $H$, Nikaidou $T$, Hayashi $H$. Detection of sentinel lymph node in gastric cancers based on immunohistochemical analysis of micrometastases. J Surg Oncol. 2004;87:32-8.

24. Nimura H, Narimiya N, Mitsumori N, Yamazaki Y, Yanaga K, Urashima M. Infrared ray electronic endoscopy combined with indocyanine green injection for detection of sentinel nodes of patients with gastric cancer. Br J Surg. 2004;91:575-9.

25. Lee JH, Ryu KW, Kim CG, Kim SK, Choi IJ, Kim YW, et al. Comparative study of the subserosal versus submucosal dye injection method for sentinel node biopsy in gastric cancer. EJSO. 2005;31:965-8.

26. Ishizaki M, Kurita A, Kubo Y, Takashima S, Nishina T, Nishimura E. Evaluation of sentinel node identification with isosulfan blue in gastric cancer. EJSO. 2006;32:191-6.

27. Lee JH, Ryu KW, Kim CG, Kim SK, Lee JS, Kook MC, et al. Sentinel node biopsy using dye and isotope double tracers in early gastric cancer. Ann Surg Oncol. 2006;13(9):1169-74.

28. Miyake K, Seshimo A, Kameoka S. Assessment of lymph node micrometastases in early gastric cancer in relation to sentinel nodes. Gastric Cancer. 2006;9:197-202.
29. Park DJ, Lee HJ, Lee HS, Kim WH, Kim HH, Lee Du, et al. Sentinel node biopsy for cT1 and cT2a gastric cancer. EJSO. 2006;32:48-54.

30. Rino Y, Takanashi Y, Harada H, Ashida A, Seki H, Yukawa N, et al. Technique and assessment of sentinel lymph node biopsy usefulness in laparoscopy-assisted distal gastrectomy. Surg Endosc. 2006;20:1887-91.

31. Tajima Y, Yamazaki K, Masuda Y, Kato M, Yasuda D, Aoki T, et al. Sentinel node mapping guided by indocyanine green fluorescence imaging in gastric cancer. Ann Surg. 2009;249(1):5862.

32. Uenosono $\mathrm{Y}$, Natsudoe S, Ehi K, Arigami T, Hokita S, Aikou T. Detection of sentinel nodes and micrometastases using radioisotope navigation and immunohistochemistry in patients with gastric cancer. Br J Surg. 2005;92:886-9.

33. Mochiki E, Kuwano H, Kamiyama Y, Aihara R, Nakabayashi T, Katoh $\mathrm{H}$, et al. Sentinel lymph node mapping with technetium$99 \mathrm{~m}$ colloidal rhenium sulphide in patients with gastric carcinoma. Am J Surg. 2006;191:465-9.

34. Yanagita S, Natsugoe S, Uenosono Y, Kozono T, Ehi K, Arigami $\mathrm{T}$, et al. Sentinel node micrometastases have high proliferative potential in gastric cancer. J Surg Res. 2008;145:238-43.

35. Lee YJ, Ha WS, Park ST, Choi SK, Hong SC, Park JW. Which biopsy method is more suitable between a basin dissection and pick-up biopsy for sentinel nodes in laparoscopic sentinel-node navigation surgery (LSNNS) for gastric cancer? J Laparoendosc Adv Surg Tech. 2008;18(3):357-63.

36. Aikou T, Higashi H, Natsugoe S, Hokita S, Baba M, Tako S. Can sentinel node navigation surgery reduce the extent of lymph node dissection in gastric cancer? Ann Surg Oncol. 2001;8(9 Sup$\mathrm{pl}): 90 \mathrm{~S}-3 \mathrm{~S}$.

37. Jamieson JK, Dobson JF. The lymphatic system of the stomach. Lancet. 1907;169:1061-6.

38. Coller FA, Kay EB, Mclntyre RS. Regional lymphatic metastases of carcinoma of the stomach. Arch Surg. 1941;43:748-61.

39. Raabe A, Beck J, Gerlach R, Zimmermann M, Seifert V. Nearinfrared indocyanine green video angiography: a new method for intraoperative assessment of vascular flow. Neurosurgery. 2003;52:132-9.

40. Takayama S, Furuhama K, Ohura K, Onodera T, Akimoto T. Experimental studies on the usefulness of indocyanine green (ICG) as a lymphatic vital dye (in Japanese). Oyo Yakuri/Pharmacometrics. 1980;19:603-14.

41. McMasters KM, Tuttle TM, Carlson DJ, Brown CM, Noyes RD, Glaser RL, et al. Sentinel lymph node biopsy for breast cancer: a suitable alternative to routine axillary dissection in multi-institutional practice when optimal technique is used. J Clin Oncol. 2000;18(13):2560-6.

42. Valsecchi ME, Silbermins D, de Rosa N, Wong SL, Lyman GH. Lymphatic mapping and sentinel lymph node biopsy in patients with melanoma: a meta-analysis. J Clin Oncol. 2011;29(11): 1479-87. 